\title{
OBSERVACIONES SOBRE LA GERMINACIÓN DE TRES ESPECIES DEL GÉNERO Citharexylum Jacq. EMPLEADAS EN RESTAURACIÓN ECOLÓGICA
}

\section{Observations on the germination of three species of Citharexylum Jacq. used for ecological restoration}

Palabras clave: Citharexylum, semillas, Verbenaceae, pireno

Key words: Citharexylum, seeds, Verbenaceae, pyrene

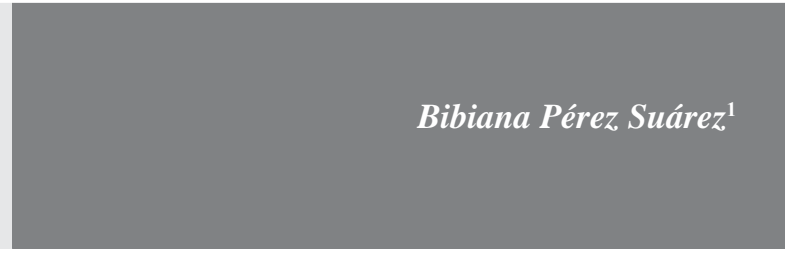

\section{RESUMEN}

Cada vez es mayor la necesidad de contar con material vegetal de buena calidad que permita desarrollar proyectos de restauración ecológica a gran escala. Por este motivo se priorizaron tres especies propias del bosque alto andino: Citharexylum montanum, Citharexylum subflavescens y Citharexylum sulcatum. Con el fin estudiar su germinación, los pirenos de estas especies fueron tratados previamente a la germinación utilizando escarificación e hidratación, hidratación por 96 horas e hidratación por 72 horas respectivamente en cada especie mencionada. En las semillas de cada especie se evaluó el porcentaje total de germinación, día de inicio de la germinación y el tiempo medio de germinación (MGT). El porcentaje de germinación total fue bajo para todas las especies; siendo C. subflavescens la especie que presentó el valor más alto $(41,3 \%$;). Así mismo, solamente el tratamiento de escarificación e inmersión realizado a la especie C. montanum favoreció el porcentaje de germinación. El menor tiempo de inicio de la germinación fue de 20 días para las especies $C$. subflavescens y C. montanum. En general las tres especies presentaron una germinación heterogénea, por este motivo el índice de tiempo medio de germinación (MGT) fue alto para todas las especies. Las observaciones realizadas sugieren que la escarificación física previa a la hidratación favore- ce tanto el porcentaje, como el día de inicio de la germinación en Citharexylum.

\section{ABSTRACT}

High-quality plant material is needed to allow the development of large-scale projects in ecological restoration. For this reason, three native species from the Andean forest were prioritized to study their germination: Citharexylum montanum, Citharexylum sulcatum and Citharexylum subflavescens. Pyrenes of these species were subjected to different pre-germination methods such as: scarification and hydration, hydration for 96 hours and hydration for 72 hours respectively for each species mentioned. The total percentage of germination, day of initiation of germination and mean germination time (MGT) were assessed for each species. As a result, total germination percentage was low for all species; C. subflavescens was the specie that presented the highest value $(41.3 \%)$. Only the treatment of scarification and hydration applied to $C$. montanum favored the germination percentage. The fastest time for germination initiation was 20 days for the species C. subflavescens and C. montanum. Due to the fact that the three species showed heterogeneous germination, the mean germination time (MGT) was high for all species. The observations suggest that physical scarification prior to hydration promotes

\footnotetext{
Grupo de Restauración Ecológica; Subdirección Científica, Jardín Botánico de Bogotá José Celestino Mutis. Bogotá, Colombia, bibiforest2006@ gmail.com
} 
both the percentage and the day of initiation of germination in Citharexylum.

\section{INTRODUCCIÓN}

Las investigaciones realizadas en el tema de restauración ecológica en bosques tropicales han identificado principalmente tres factores que limitan la regeneración: 1) la carencia de dispersión o de propágulos en el banco de semillas, 2) bajas tasas de germinación y 3) bajas tasas de reclutamiento debido a los efectos negativos de las enfermedades, depredación y competencia, o condiciones físicas adversas (Meli 2003). En ese sentido, para ayudar al ecosistema a acelerar los procesos de sucesión natural, se viene implementando la plantación de individuos propios de las áreas a restaurar.

Existe poco conocimiento acerca de la reproducción de especies nativas con fines de restauración ecológica (Castañeda et al. 2007) y cada vez es mayor la necesidad de contar con material vegetal de buena calidad que permita desarrollar proyectos a mayor escala. Por lo anterior uno de los aspectos que requieren mayor atención es el estudio de la propagación de especies propias de los ecosistemas a restaurar. El conocimiento sobre la germinación de especies locales es entonces, el paso inicial toda vez que el material vegetal (plántulas) es el insumo de partida con el que se cuenta en el proceso.

El presente estudio se enmarca en la línea de investigación en protocolos de propagación de especies andinas priorizadas con fines de restauración, del proyecto de restauración, rehabilitación y/o recuperación ecológica de áreas alteradas en el Distrito Capital y la Región, desarrollada por la Subdirección Científica del Jardín Botánico José Celestino Mutis (JBB). Dentro de esta línea de investigación se seleccionaron tres especies nativas del género Citharexylum, ampliamente utilizadas por el proyecto en las zonas a restaurar. Las especies seleccionadas fueron Citharexylum montanum Moldenke, Citharexylum subflavescens Blacke y Citharexylum sulcatum Moldenke. Algunas de las características que hacen interesantes estas especies para su uso en proyectos de restauración ecológica son: especies melíferas, los frutos son fuente alimenticia para la avifauna, la madera es utilizada para leña y postes para cerca. Así mismo se registran usos para barreras cortavientos, recuperación ambiental y ornamento por sus coloridos frutos, además de presentar rápido crecimiento (Foster \& Janson 1985, Zanon et al. 1997, Mahecha et al. 2004, Wehmuth et al. 2007). En C. subflavescens se presenta un crecimiento de hasta $2.5 \mathrm{~m}$ por año (Vargas 2002).

Las unidades de germinación del género Citharexylum se conocen con el nombre de pirenos, están conformados por un endocarpio grueso de consistencia leñosa, muy resistente a la perforación o quiebre. Los frutos del genero Citharexylum contienen dos pirenos, cada uno de los cuales posee dos lóculos y en cada lóculo una semilla (Woodson et al 1973). Los pirenos en la especie C. montanum alcanzan $2 \mathrm{~mm}$ de espesor. Dentro de cada pireno se encuentran dos semillas fuertemente adheridas, de tal forma que las semillas no pueden ser extraídas sin sufrir daño mecánico. Así mismo, los pirenos presentan dos opérculos de gran tamaño por donde sale la radícula durante el inicio de la germinación.

Se conoce que las semillas de algunas especies de Citharexylum son ortodoxas, ya que pueden perder humedad sin disminuir el poder germinativo $\mathrm{y}$ permanecer en esta condición durante varios meses (Zanon et al. 1999).

En este trabajo se estudió el efecto de la utilización de tratamientos pregerminativos en el comportamiento germinativo de las especies mencionadas.

\section{MATERIALES Y MÉTODOS}

\section{OBTENCIÓN Y MANEJO DEL MATERIAL VEGETAL}

Los frutos de las especies C. montanum y C. subflavescens se colectaron de individuos localizados en la colección viva del JBB. Los frutos de la especie C. sulcatum fueron colectados en la 
localidad de Ciudad Bolívar (Vereda Pasquilla), al suroccidente de Bogotá. Se cosecharon frutos maduros y sanos.

Los pirenos se extrajeron de frutos recién cosechados y secados a la sombra por dos días (Iossi et al. 2006), cuya pulpa se dejó descomponer por una semana. Los pirenos se extrajeron manualmente macerando los frutos y separando la pulpa mediante el roce contra un tamiz; y retirando los restos de fruto mediante lavado.

\section{ENSAYOS DE GERMINACIÓN}

Se aplicaron 3 tratamientos pregerminativos frecuentemente utilizados en el proceso de propagación masiva de dichas especies en el JBB (Tabla 1). Se empleó un tratamiento pregerminativo en cada especie y se utilizaron 4 replicas por tratamiento cada una compuesta por 50 pirenos. Los testigos de cada especie no recibieron tratamiento pregerminativo

Tabla 1. Tratamientos pregerminativos aplicados en pirenos de Citharexylum

\begin{tabular}{lcc}
\hline Especie & $\begin{array}{c}\text { Tratamiento } \\
\text { pregerminativo }\end{array}$ & $\begin{array}{c}\text { Tiempo de } \\
\text { almacenamiento }\end{array}$ \\
\hline C. montanum & $\begin{array}{c}\text { Escarificación por medio } \\
\text { de lija e hidratación por } \\
24 \text { horas }\end{array}$ & 10 días \\
C. subflavescens & Hidratación por 96 horas & 23 días \\
C. sulcatum & Hidratación por 72 horas & 35 días \\
\hline
\end{tabular}

Teniendo en cuenta que cada semilla se encuentra fuertemente adheridas al pireno (Zanon et al. 1997), estos no fueron divididos para individualizar las semillas, pero si se tuvo en cuenta el número de semillas dentro de él. Los pirenos se desinfestaron con hipoclorito de sodio al 3\% durante 10 minutos. Posteriormente, los pirenos se secaron al aire libre durante dos días, se almacenaron en bolsas plásticas de cierre hermético a temperatura ambiente $\left(18^{\circ} \mathrm{C}^{+/-}\right.$ 3) y humedad relativa de $42.58 \%$ hasta el momento de la siembra.
La siembra se realizó en bandejas de germinación sin alveolos y con tapa transparente, en un medio de propagación compuesto por sustrato orgánico (tierra negra), arena y cascarilla en proporción 3:1:1 en volumen, los cuales fueron previamente pasteurizados, para este procedimiento se utilizó un horno pasteurizador donde se sometieron los sustratos a una temperatura de $120^{\circ} \mathrm{C}$ en seco durante 3 horas. Los pirenos se sembraron a $5 \mathrm{~mm}$ de profundidad dejando entre ellos una distancia de $2 \mathrm{~cm}$, las bandejas se regaron una vez por semana.

Los ensayos se realizaron en el laboratorio e invernadero del Jardín Botánico José Celestino Mutis a una temperatura entre $17.1^{\circ} \mathrm{C}$ y $20.1^{\circ} \mathrm{C}$ con un promedio de $18^{\circ} \mathrm{C}$ y a una humedad relativa del $100 \%$, localizado a $2650 \mathrm{~m}$ de altitud en la zona urbana de Bogotá.

\section{ANÁLISIS DE DATOS}

Siguiendo la metodología empleada por Laskowski \& Bautista (2002), se consideró la semilla germinada cuando el pireno emergió a la superficie del sustrato y dejó ver parte del hipocótilo. Cada 4 días se registró el número de semillas germinadas y con estos datos se obtuvo el porcentaje total de germinación, el día de inicio y el índice del tiempo medio de germinación (MGT). El MGT se calculó utilizando la fórmula de Guariguata \& Azocar (1998):

$$
\operatorname{MGT}=\sum\left(\mathbf{N}_{\mathrm{i}} \mathbf{x} \mathbf{T}_{\mathrm{i}}\right) / \sum \mathbf{N}_{\mathrm{i}}
$$

Donde: MGT=Índice de tiempo medio de germinación

$$
\begin{aligned}
& \mathbf{N}=\text { germinación total en un tiempo } \mathrm{T} \\
& \mathbf{T}=\text { tiempo en días }
\end{aligned}
$$

Para cada una de las especies, se compararon las medias de las variables germinación total, día de inicio de germinación y MGT entre el tratamiento y el testigo; mediante la prueba t para muestras independientes. En aquellos casos en que los datos 
no cumplían los supuestos de normalidad (ShapiroWilk) y/o homocedasticidad (Levene) se realizó la prueba de comparación de medianas de U MannWhitney.

Entre especies se comparó el porcentaje de germinación total y el MGT de semillas no tratadas (testigos) mediante un análisis de Kruskal-Wallis y el día de inicio de germinación mediante análisis de varianza. Los mejores resultados de tiempo mínimo de germinación se establecieron con la prueba de rangos múltiples de Bonferroni. Todas las pruebas estadísticas fueron realizadas utilizando el programa estadístico STATGRAPHICS Centurion XV versión 15.02.06 (StatPoint, Inc 2007).

\section{RESULTADOS}

Los frutos de las especies de Citharexylum estudiadas son drupas globosas semi-esféricas de color rojo intenso cuando están maduros para el caso de C. montanum y C. subflavescens y violeta oscuro para C. sulcatum, con mesocarpio carnoso y blando, epicarpio glabro y semilustroso.

\section{EFECTO DE LOS TRATAMIENTOS PREGERMINATIVOS}

En C. montanum el porcentaje de germinación fue $18.5 \%$ mayor en las semillas escarificadas en comparación con aquellas notratadas $\left(\mathrm{t}_{(0.05 ; 3)}=3.101\right.$; $\mathrm{p}=0.028)$. La germinación total alcanzó $39 \%$ y $20.5 \%$ el día 75 para el tratamiento y el testigo. Por el contrario el tiempo de inicio de la germinación (19 días) $\left(\mathrm{t}_{(0.05: 3)}=-0.696 ; \mathrm{p}=0.512\right)$, ni el tiempo medio de germinación (MGT) se afectaron con el tratamiento de escarificación $\left(\mathrm{U}_{(0.05 ; 3)}=<0.05 ; \mathrm{p}=\right.$ 0.885). El MGT de las semillas tratadas fue de 68.2 días y 69.1 días para el testigo. La distribución de los valores de germinación total, se muestran en la figura 1.

La germinación acumulada presenta un comportamiento sigmoidal, donde se aprecia un fuerte incremento en la pendiente a partir del día 29 hasta el día 40 en el tratamiento que tiende a

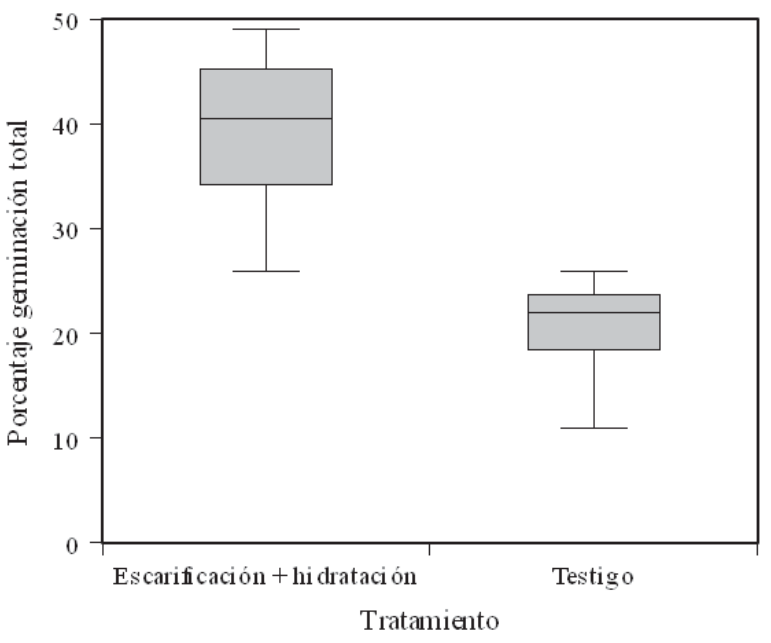

Figura 1. Porcentaje de germinación en la especie Citharexylum montanum

estabilizarse después del día 68 (Figura 2). En la gráfica de germinación diaria se observa que existe un pico de germinación en el periodo comprendido entre los días 29 y 40 para el tratamiento.

En la especie C. subflavescens el tratamiento de inmersión no favoreció el porcentaje de germinación $\left(\mathrm{t}_{(0.05 ; 3)}=0.237 ; \mathrm{p}=0.820\right)$, ni el día de inicio de germinación $\left(\mathrm{t}_{(0.05 ; 3)}=1.732 ; \mathrm{p}=0.133\right)$ o el tiempo medio de germinación $\left(\mathrm{t}_{(0.05 ; 3)}=1.300 ; \mathrm{p}=0.241\right)$. Un $40.6 \%$ de las semillas hidratadas germinaron en comparación con $41.3 \%$ del testigo, iniciando a los 20 días en los dos casos y alcanzando un valor de MGT de 52 días en ambos casos.

La germinación de C. subflavescens se presentó de una manera rápida; la curva presenta una fuerte pendiente desde el inicio hasta el día 40 donde tiende a estabilizarse, igualmente se observa un pico en la gráfica de germinación diaria que coincide con este periodo (Figura 3).

En C. sulcatum el tratamiento pregerminativo no mejoró el porcentaje de germinación $\left(\mathrm{U}_{(0.05 ; 3)}=-3.5\right.$; $\mathrm{p}=0.524)$, ni el día de inicio de la germinación $\left(\mathrm{t}_{(0,05 ; 3)}\right.$ $=0.568 ; \mathrm{p}=0.585)$. El porcentaje de germinación alcanzó $24.4 \%$ en el tratamiento y $26.6 \%$ en el testigo. El inicio de la germinación se presentó el día 52 desde la siembra. Por el contrario, el tiempo medio de germinación fue menor en el tratamiento 


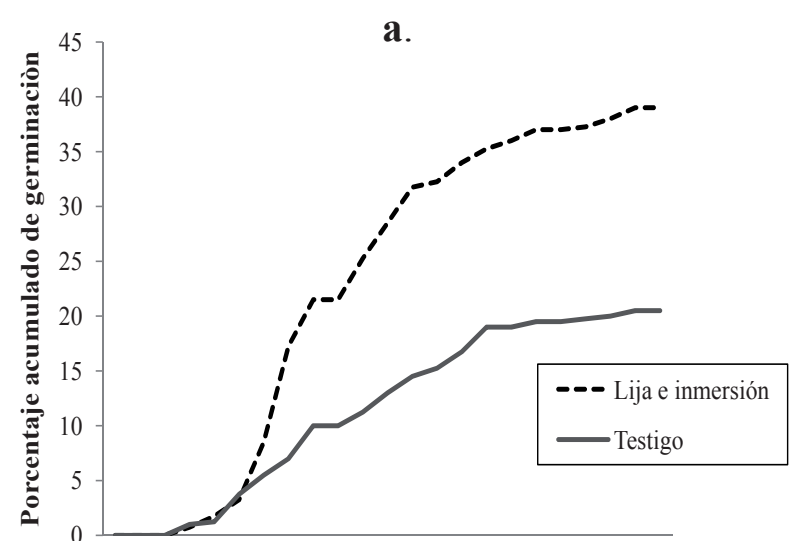

$\begin{array}{llllllllllll}4 & 12 & 22 & 29 & 40 & 48 & 60 & 68 & 75 & 82 & 88 & 101\end{array}$

Días

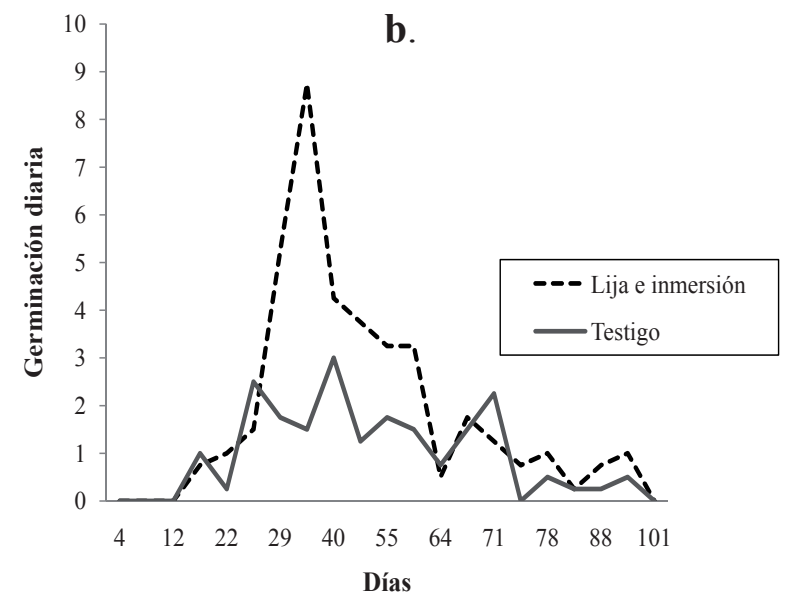

Figura 2. Curvas de germinación en Citharexylum montanum a. Porcentaje acumulado de germinación. b. germinación diaria.

que en el testigo, es decir que el tratamiento afecto significativamente el MGT $\left(\mathrm{t}_{(0.05 ; 3)}=3.002 ; \mathrm{p}=\right.$ 0.017)(Figura 4).

Para esta especie las curvas de germinación acumulada presentan un pendiente débil entre los días 50 y 100. Así mismo, la germinación diaria no se presenta de forma homogénea, es lenta y variable y no tendió a estabilizarse en el periodo observado (Figura 5).

\section{COMPARACIÓN DE LA GERMINACIÓN ENTRE ESPECIES}

Al comparar la germinación de las semillas no tratadas de las tres especies se encontró que el porcentaje de germinación fue significativamente

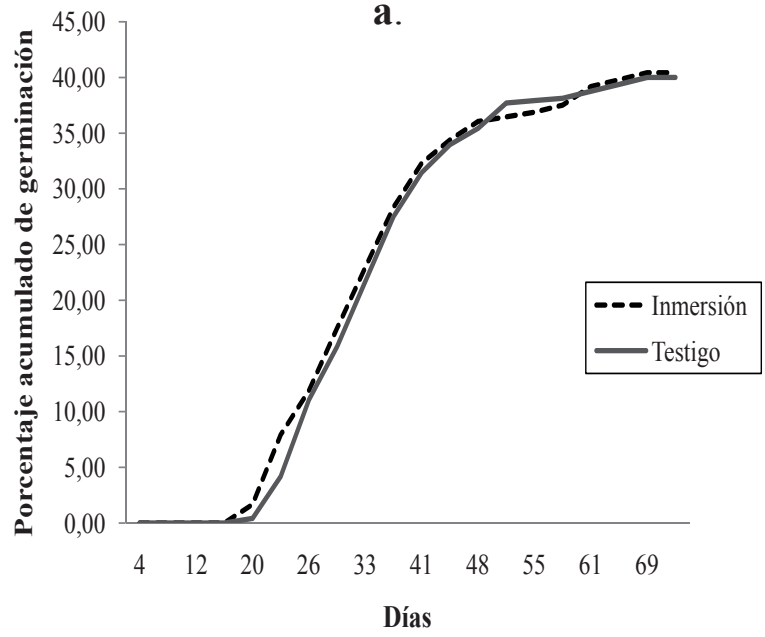

b.

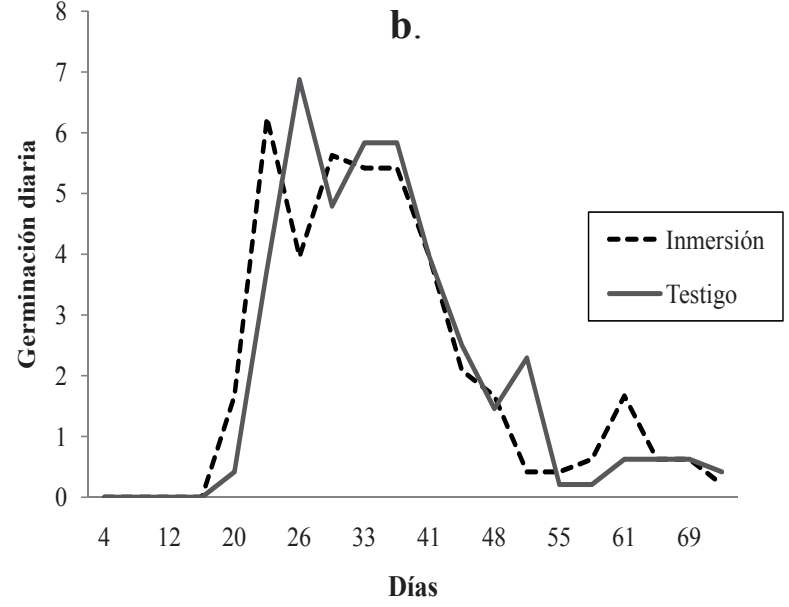

Figura 3. Curvas de germinación en Citharexylum subflavescens a. Porcentaje acumulado de germinación. b. germinación diaria.

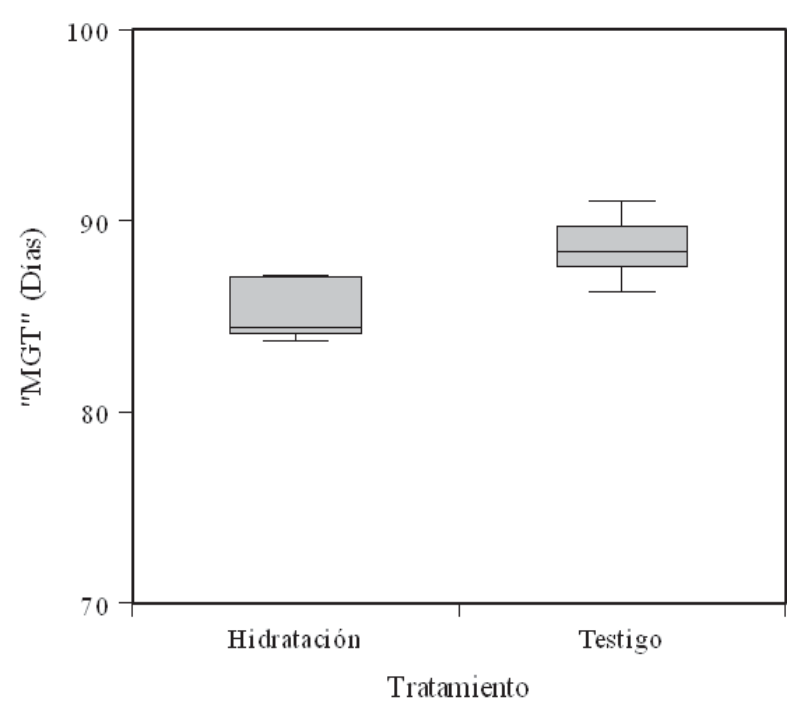

Figura 4.Tiempo medio de germinación (MGT) en la especie Citharexylum sulcatum 

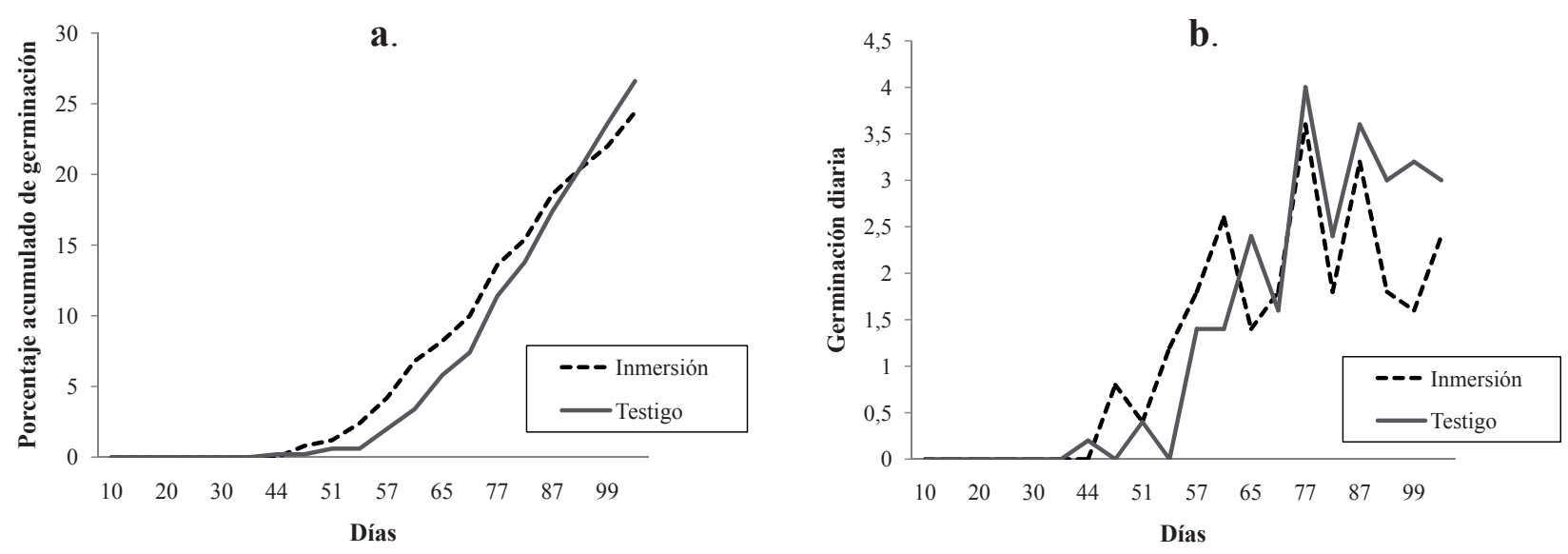

Figura 5. Curvas de germinación en Citharexylum sulcatum a. Porcentaje acumulado de germinación. b. germinación diaria.

mayor para la especie C. subflavescens $\left(\mathrm{H}_{(0.05 ; 2)}=\right.$ 9.295; $\mathrm{p}=0.009)$, de la misma manera el tiempo medio de germinación fue menor para esta especie $\left(\mathrm{H}_{(0.05 ; 2)}=10.681 ; \mathrm{p}=0.004\right)$, mientras que el día de inicio fue significativamente menor para $C$. sulcatum $\left(\mathrm{F}_{(0.05 ; 2)}=72.840 ; \mathrm{p}=<0.05\right)$.

\section{DISCUSIÓN}

La información sobre el comportamiento germinativo de las especies de Citharexylum nativas del bosque andino colombiano presentada en este estudio aunque preliminar es pionera dado que se trata de especies muy valiosas pero poco estudiadas. Es de anotar que los frutos y semillas analizados no fueron colectados directamente de poblaciones naturales de las especies.

Las semillas de las tres especies de Citharexylum utilizados en este estudio presentaron en general bajos porcentajes de germinación, siendo $C$. subflavescens la que presentó los valores más altos con un promedio de $41.3 \%$ en el testigo. Se observó que el tratamiento de escarificación física por medio de lija y la posterior hidratación por 24 horas favoreció significativamente la germinación en la especie C. montanum, mientras que los tratamientos que solo incluían hidratación, que se aplicaron a las otras dos especies no mejoraron la germinación, posiblemente debido a que los pirenos de estas especies presenten algún grado de impermeabilidad al agua. Laskowski \& Bautista
(2002), estudiaron el efecto de la escarificación en semillas de otra especie de Verbenácea, con características similares a la de las semillas de las especies estudiadas y demostraron que la escarificación de las semillas presentó un ligero aumento en el porcentaje de germinación.

El día de inicio de germinación ocurrió en un periodo de 20 días para las especies C. montanum y C. subflavescens, mientras que para $C$. sulcatum demoró 52 días en iniciar, es decir más del doble del tiempo que les llevó a las otras dos especies iniciar el proceso; además esta ultima especie presentó los porcentajes más bajos de germinación total y el mayor índice de tiempo medio de germinación, MGT. Aun cuando se conoce que algunas especies de Citharexylum son ortodoxas $\mathrm{y}$ pueden permanecer viables en almacenamiento durante un periodo prolongado, los resultados observados en las especies de estudio pueden deberse a las condiciones de almacenamiento y a diferencias en el tiempo transcurrido desde la obtención de las semillas hasta el momento de la siembra, el cual fue de 35 días para C. sulcatum mientras que las otras especies fueron sembradas en un periodo de tiempo menor; es posible que las semillas de esta especie hayan perdido viabilidad durante el almacenamiento y por este motivo se hace evidente la necesidad de adelantar estudios sobre el almacenamiento de semillas. Es necesario anotar que los métodos de escarificación y los tiempos de almacenamiento previo a la siembra fueron diferentes entre las especies y se desconoce 
como esto pudo haber afectado el comportamiento germinativo de las diferentes especies.

\section{CONCLUSIONES}

Las observaciones de este estudio sugieren que métodos de escarificación física como el lijado de los pirenos previo al tratamiento de hidratación resulta favorable para mejorar la germinación de semillas de por lo menos una especie de Citharexylum. Por lo anterior, se sugiere profundizar en investigaciones sobre tratamientos pregerminativos que contemplen el uso de la escarificación física del endocarpio así como para conocer el correcto almacenamiento para las semillas de estas especies.

\section{AGRADECIMIENTOS}

A los profesionales de la Subdirección Científica del Jardín Botánico José Celestino Mutis especialmente al grupo Coordinador del Proyecto de Restauración Ecológica por sus oportunas sugerencias en la marcha de la investigación, a Catalina Azula por su revisión del resumen en inglés, a Héctor Felipe Ríos por la revisión final del texto, a Héctor Lancheros y Oscar Rojas por su colaboración con el análisis de datos y revisión final del texto.

\section{REFERENCIAS BIBLIOGRÁFICAS}

Castañeda, S., A. Garzón, M. Cantillo, M. Torres \& L. Silva. 2007. Análisis de la respuesta de ocho especies nativas del bosque altoandino ante dos métodos de propagación. Revista Colombia Forestal 10(20): 79-90.

Foster, S. \& C. Janson. 1985. The relationship between seed size and establishment conditions in Tropical Woody Plants. Ecological Society of America 66(3): 773-778.

Guariguata, M. \& A. Azocar. 1988. Seed Bank Dynamics and Germination Ecology in Espele- tia timotensis (Compositae), an Andean Giant Rosette. Biotropica 20(1): 54-59.

Iossi, E., F. Vitti \& R. Sader. 2006. Anatomia e germinação de sementes de Phoenix roebelenii O'Brien (Arecaceae). Revista Brasileira de Sementes 28(3): 121-128

Laskowski, L. \& D. Bautista. 2002. Efecto de la escarificación y profundidad de siembra sobre la germinación y emergencia de Malpighia emarginata DC. Bioagro 14(2): 77-38

Mahecha, G., A. Escobar, D. Camelo, M. Rozo \& D. Barrero. 2004. Vegetación del territorio CAR, 450 especies de sus llanuras y montañas. Corporación Autónoma Regional de Cundinamarca (CAR). Bogotá. 871 p.

Meli, P. 2003. Restauración ecológica de bosques tropicales. Veinte años de investigación académica. INCI 28(10): 581-589.

StatPoint, Inc. 2005. STATGRAPHICS Centurion XV versión 15.02.06.

Vargas, W. 2002. Guía ilustrada de las plantas de las montañas del Quindío y los Andes centrales. Universidad de Caldas. Manizales. $813 \mathrm{p}$.

Wehmuth, E., R. Pescador, S. luiz \& A. Uhlmann. 2007. Germinaçâo de Citharexylum myrianthum Cham. (Verbenaceae) em Diferentes Substratos. Revista Brasileira de Biociências 5(2): 741-743

Woodson, R., R. W. Schery \& H. N. Moldenke. 1973. Flora of Panama. Part IX. Family 168. Verbenaceae. Annals of the Missouri Botanical Garden 60(1): 92-93

Zanon, A., A. Aparecido \& J. Pereira. 1997. Germinação em laboratório e armazenamento de sementes de tarumã-branco (Citharexylum myrianthum Cham.). Boletin de Pesquisa Florestal, Colombo 35:75-82 